\title{
Severe acute kidney injury in a 3-year-old boy with fever and pleural effusion: Questions
}

\author{
Aakash Chandran Chidambaram ${ }^{1} \cdot$ Sriram Krishnamurthy ${ }^{1}$ (D) $\cdot$ Bobbity Deepthi ${ }^{1} \cdot$ Pediredla Karunakar $^{1}$. \\ Kaushik Maulik ${ }^{1}$ - Sreeram Chandra Murthy Peela ${ }^{2} \cdot$ Sujatha Sistla ${ }^{2} \cdot$ Sree Rekha Jinkala ${ }^{3}$
}

Received: 14 April 2020 / Revised: 15 April 2020 / Accepted: 22 April 2020 / Published online: 28 May 2020

(C) IPNA 2020

\section{Case report}

A 3-year-old boy presented with a history of fever and cough for 6 days, which was associated with anorexia. There was no past history of lower respiratory tract infections or hospitalizations. The family history was unremarkable for any infections, hospitalizations, or major illnesses. There was no history of any drug intake for the child other than paracetamol for fever. The boy had received vaccines as per the national immunization program of India, but not the pneumococcal vaccine, varicella, or influenza vaccines. The child was conscious but had increased work of breathing at presentation to our hospital. He was developmentally normal for age, and the weight $(14 \mathrm{~kg} ;-0.24 \mathrm{z})$ and height $(95 \mathrm{~cm} ;-0.38 \mathrm{z})$ were within acceptable limits. Physical examination revealed axillary temperature $38.5^{\circ} \mathrm{C}$, heart rate $140 / \mathrm{min}$, respiratory rate $51 /$ min (with significant subcostal and intercostal retractions), blood pressure $90 / 55 \mathrm{mmHg}$, and capillary refill time $3 \mathrm{~s}$. The oxygen saturation by pulse oximetry was $85 \%$ on room air. Reduced air entry in the left infra-mammary, infra-scapular, and infra-axillary areas, with reduced vocal fremitus and reduced vocal resonance, were noted. Fine crepitations were also noted in these areas. The central nervous system examination was also normal, with no neck rigidity, and Kernig's

The answers to these questions can be found at https://doi.org/10.1007/ s00467-020-04591-7.

Sriram Krishnamurthy

drsriramk@yahoo.com

1 Department of Pediatrics, Jawaharlal Institute of Postgraduate Medical Education and Research (JIPMER), Pondicherry 605006, India

2 Department of Microbiology, Jawaharlal Institute of Postgraduate Medical Education and Research (JIPMER), Pondicherry, India

3 Department of Pathology, Jawaharlal Institute of Postgraduate Medical Education and Research (JIPMER), Pondicherry, India sign and Brudzinski's sign were negative. There were no focal neurological deficits. The other systemic examinations were unremarkable.

Supplemental oxygen by mask and intravenous (IV) fluids were administered, and he was admitted to the pediatric intensive care unit (PICU). The chest X-ray revealed left-sided pleural effusion (Fig. 1). He was started on intravenous ceftriaxone and vancomycin, after obtaining necessary blood samples for investigations (Table 1). Blood investigations revealed neutrophilic leukocytosis. Serum creatinine was $0.41 \mathrm{mg} / \mathrm{dL}$ at this juncture. A diagnostic pleural tap was done after which intercostal drainage tube was inserted (Fig. 1). The pleural fluid was sent for gram stain, bacterial culture, and molecular diagnostic tests. Nasopharyngeal swabs for influenza A (including H1N1) and influenza B were negative by realtime polymerase chain reaction (RT-PCR). Other investigational workup for pleural effusion, e.g., IgM immunofluorescence for scrub typhus, microscopic agglutination test for leptospirosis, and Widal test (for enteric fever), were negative. Lumbar puncture was done and meningitis was ruled out. Urinalysis did not show any abnormalities. After $24 \mathrm{~h}$ of admission, there was worsening respiratory distress with oxygen desaturation and continued absent air entry on the left side. The child required mechanical ventilation. The blood culture and pleural fluid cultures were negative for any organism.

By the 3rd day of admission, the child had a sudden decline in urine output progressing to complete anuria. This was associated with a sudden drop in hemoglobin from 10.1 to $6.5 \mathrm{~g} /$ $\mathrm{dL}$ with thrombocytopenia $(60,000 / \mu \mathrm{L})$, without any evidence of ongoing blood loss (Table 1). Peripheral smear examinations for schistocytes and reticulocyte count were performed. Serum lactate dehydrogenase (LDH) levels were found to be elevated (4049 U/L). Serum complement levels were normal [C3-94 mg/dL (reference range $80-140 \mathrm{mg} / \mathrm{dL}$ ); C4-24 mg/ dL (reference range 12-42 mg/dL)] and direct Coombs test (DCT) was positive. The serum aspartate transaminase (AST) and alanine transaminase (ALT) were high (545 U/L and 
Fig. 1 a Chest X-ray revealed left-sided pleural effusion. $\mathbf{b}$ Diagnostic pleural tap after which intercostal drainage tube was inserted
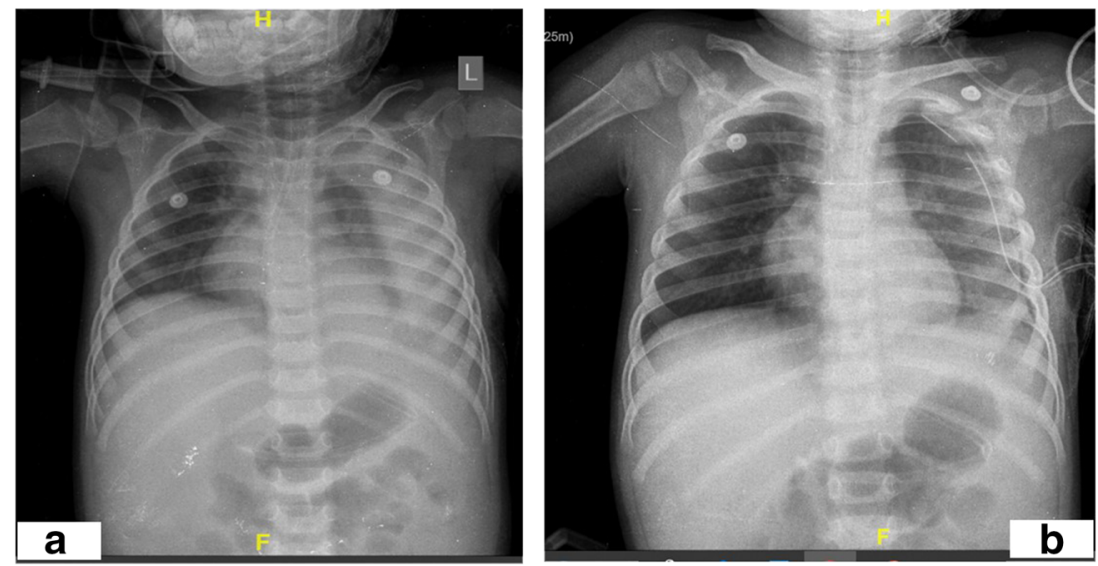

Table 1 Blood investigations during hospital stay

\begin{tabular}{|c|c|c|c|c|c|}
\hline Laboratory parameter & $\begin{array}{l}\text { Day } 1 \text { of hospital } \\
\text { stay }\end{array}$ & $\begin{array}{l}\text { Day } 3 \text { of hospital } \\
\text { stay }\end{array}$ & $\begin{array}{l}\text { Day } 5 \text { of hospital } \\
\text { stay }\end{array}$ & $\begin{array}{l}\text { Day } 9 \text { of hospital } \\
\text { stay }\end{array}$ & Normal/reference values \\
\hline Hemoglobin (g/dL) & 10.1 & 6.5 & 3.5 & 8.0 & $>11.5$ \\
\hline $\begin{array}{l}\text { White blood cell count } \\
\quad(\text { cells } / \mu \mathrm{L})\end{array}$ & 16,200 & 13,750 & 23,320 & 56,350 & $4500-11,000$ \\
\hline Platelet $($ cells $/ \mu \mathrm{L})$ & 165,000 & 60,000 & 134,000 & 81,000 & $150,000-450,000$ \\
\hline Urea $(\mathrm{mg} / \mathrm{dL})$ & 42 & 262 & 254 & 187 & $20-40$ \\
\hline Creatinine (mg/dL) & 0.41 & 3.05 & 3.72 & 3.14 & $\begin{array}{l}\text { Normal eGFR for this age was expected } \\
\text { to be at least } 90 \mathrm{ml} / \mathrm{min} / 1.73 \mathrm{~m}^{2}\end{array}$ \\
\hline $\begin{array}{l}\text { Lactate dehydrogenase } \\
\text { (LDH) (IU/L) }\end{array}$ & & 4049 & 5572 & 3960 & $150-450$ \\
\hline
\end{tabular}

$166 \mathrm{U} / \mathrm{L}$, respectively). The d-dimer and fibrin degradation products (FDP) were negative, and the prothrombin time (PT) and activated partial thromboplastin time (aPTT) were normal. Repeat urinalysis could not be performed, as the child was completely anuric.

Peritoneal dialysis was initiated in view of anuria and washed packed red blood cells were transfused. Despite the ongoing renal replacement therapy (which was transited to sustained low efficiency dialysis (SLED) due to the development of shock), continued mechanical ventilation, and intravenous antibiotics, the child showed no improvement in the ventilator settings and progressed to acute respiratory distress syndrome (ARDS). He subsequently developed catecholamine refractory septic shock, altered sensorium, and remained completely anuric throughout the further course of illness. He died on day 10 of hospital stay. Postmortem biopsy of the kidney was performed (Fig. 2).
Fig. 2 a, b Postmortem biopsy of the kidney
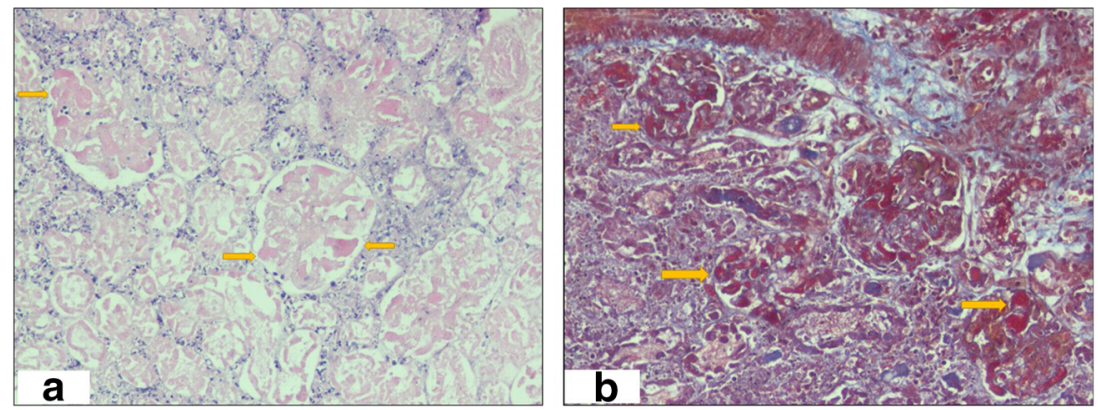


\section{Questions}

1. What are the differential diagnoses of AKI in this case?

2. What are the strategies for the management of AKI in this case? What is the role of performing complement studies and treating with plasma exchanges in this patient?

3. What could be the reason for complete anuria in this child? What are the histopathological findings in Fig. 2?

Authors' contributors Aakash Chandran Chidambaram, Sriram Krishnamurthy, Bobbity Deepthi, Pediredla Karunakar, and Kaushik Maulik managed the patient, reviewed the literature, and drafted the manuscript. Sriram Krishnamurthy critically revised the manuscript. Sreeram
Chandra Murthy Peela and Sujatha Sistla interpreted the microbiological reports. Sree Rekha Jinkala interpreted the histopathological findings. All authors contributed to reviewing of literature and drafting of the manuscript and approved the final version of the manuscript. Sriram Krishnamurthy shall act as the guarantor of the paper.

\section{Compliance with ethical standards}

Conflict of interest The authors declare that they have no conflicts of interest.

Publisher's note Springer Nature remains neutral with regard to jurisdictional claims in published maps and institutional affiliations. 